\title{
The Myth and Math of Dao De Ching (DDC): The Mathematical Functional Analysis*
}

\author{
WANG Tongjun \\ Beijing International Studies University (BISU), Beijing, China
}

\begin{abstract}
This paper aims to reveal the myth of Dao De Ching $(D D C)$ in terms of the mathematical functional analysis. It employs the Cartesian coordinate systems to illuminate $D D C$, in which varieties of core concepts are demonstrated vividly by means of one directional system, two- and three- dimension mechanisms, and polar scheme. The coordinate system clarifies complex and complicated relationships in hosts of Daoism terms, such as Dao and De, yin and yang, heaven, earth and human beings, nature, and non-being $(w u)$ and being $(y o u)$. Functional analysis is one of the most fundamental and essential devices in math, which can be applied to a truth of other fields.
\end{abstract}

Keywords: DDC, Dao, De, mathematical functional analysis, Cartesian coordinate systems

\section{Introduction}

A function using is one material mathematical model describing the changing of the objects. It involves sets, the dependence of the variables, constants, mappings, correspondence, expressions, equations, inequality, and formulas, all of which can build up our thinking ways. The gist of functions is motion and changes, the priority of which is the transformation from the static to the dynamic, and the shift from constants to variables. Equations focus on the static points, and inequalities are on dynamic intervals, while functions concern about the whole process of motion, the entire domain (Zhu \& Cheng, 2018, p. 11). Recognizedly, dynamic study is on the go in math, philosophy, as well as other fields.

A function $f$ is a rule that assigns to each element $x$ in a set $D$ exactly one element, called $f(x)$, in a set $E$ (Stewart, 2016, p. 2). The functional laws bridge two sets with mappings, so that every factor in one set can find its project in the other set, clarifying the relations of different variables.

There are four possible ways to represent a function: verbally (by a description in words), numerically (by a table of values), visually (by a graph), and algebraically (by an explicit formula)... But certain functions are described more naturally by one method than by another (Stewart, 2016, pp. 4-5). Sure, the verbal interpreting is one of the common ways to functional analysis. It holds true to other approaches, like tables, graphs, and formulas. However, the most common method of visualizing a function is its graph. If $f$ is a function with domain $D$, then its graph is the set of ordered pairs $\{(\mathrm{x}, \mathrm{f}(\mathrm{x})) \mid \mathrm{x} \in \mathrm{D}\} \quad$ (Stewart, 2016, p. 3). These paired points $(\mathrm{x}, \mathrm{y})$ in the Cartesian coordinate systems demonstrates $y=f(x), x$ is in the domain of $f$, and $y$ of the range.

\footnotetext{
* Acknowledgement: The author is grateful for research support from the project "Legal Translating in the Perspectives of Daoism and Mathematics" (WHCB18B001), Beijing Research Center for International Culture Exchange.

WANG Tongjun, Master of English Literature \& Master of Business Law, associate professor, School of English Language, Literature and Culture, Beijing International Studies University (BISU), Beijing, China.
} 
This paper concentrates on verbals and visualizations, Cartesian coordinate systems in particular. Math concentrates on measuring and ordering, which can be pictured with the coordinate systems.

Is Dao De Ching $(D D C)$ underlying a large amount of graphs? Are those key concepts full of variety of pictures? Can we visualize it?

\section{General Functional Analysis of DDC}

Functions arise whenever one quantity depends on another (Stewart, 2016, p. 2). As we know, a function is to define the relationships between and among quantities; one changing leads to another associated or corresponding variable moving. A function is a rule (Stewart, 2016, p. 7). Any law consists of so many rules, defining numerous relationships, which can be illustrated by means of functions in mathematics. This verse of Daoist poem contains a wealth of laws, laws of nature and humans. Cartesian coordinate system combines the geometrical graph with the algebra equations, revealing the mathematical beauty of harmony between numbers and graphs (Zhang, 2017, p. 188). Can the ideas in DDC be interpreted by means of coordinate systems? Variety of links in the Daoism will be revealed under the mechanism of numbers and graphs.

The derivative of a function of a real variable measures the sensitivity to change of the function value (output value) with respect to a change in its argument (input value). Derivatives are a fundamental tool of calculus. For example, the derivative of the position of a moving object with respect to time is the object's velocity: This measures how quickly the position of the object changes when time advances. ${ }^{1}$ Functions emphasize changes, and its derivative goes to the changing rate at a certain interval, and the term limits involved.

What are the derivatives of Dao and De? Myriads of things? The functioning of the Dao is its growing and developing, and when and where it can obtain the substantial changes.

\section{Analysis of the Daoism Based on Coordinate Systems}

It is known that the coordinate systems are based on the functions of some variables, which are interlinked with causality, such as $x$ causing $y$ changing. The dependent variable $y$ is the effect of the independent $x$, the leader $x$ creating a follower $y$. The two brothers $x$ and $y$ shaking hands are to demonstrate the connections in an event with other variables fixed. Thus, the role of the function of $x$ and $y$ is highlighted. A clear picture will demonstrate the complicated links in a certain event of a set with the assistance of the coordinate system. The concept of Cartesian coordinates generalizes to allow axes that are not perpendicular to each other, and/or different units along each axis. In that case, each coordinate is obtained by projecting the point onto one axis along a direction that is parallel to the other axis (or, in general, to the hyperplane defined by all the other axes). ${ }^{2}$ Generally, the horizontal axis $x$ with its projects generates another project in the axis $y$, reflecting the function of $x$, i.e., $y=f(x)$.

Mathematics already underlies many of society's most-cherished political and social inventions: ideas about cause and effect, fairness and justice, selfishness and cooperation, balancing risks, spending on welfare or national defense, and even the nature of scientific discovery itself (Cole, 1999, p. 6). There are various similar expressions in $D D C$ on the above mentioned thoughts, all of which can be illustrated with Descartes cooperated systems.

\footnotetext{
${ }^{1}$ Derivative, Wikipedia, https://en.wikipedia.org/wiki/Derivative.

${ }^{2}$ Cartesian coordinate system, Wikipedia, https://en.wikipedia.org/wiki/Cartesian_coordinate_system.
} 


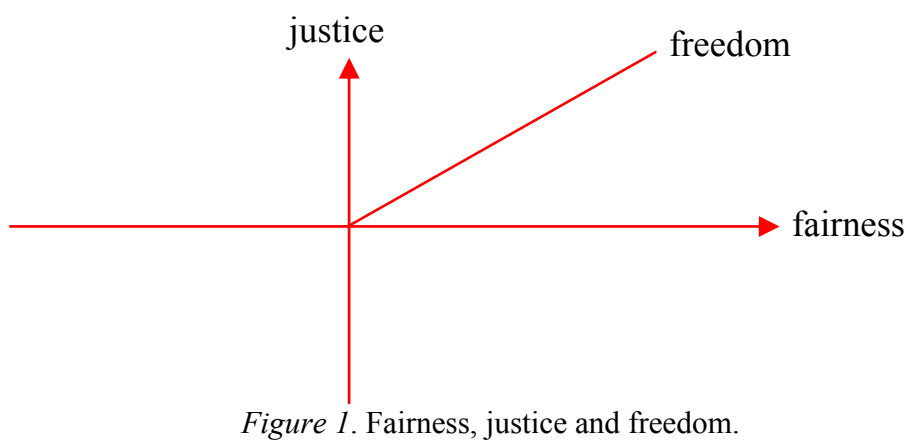

This scheme shows that freedom is the mix of fairness and justice, the dependent variable justice following the independent variable fairness, of which freedom is born. Being free is the ideal status in the reality; without fair circumstances or justice, there would be no freedom. In the Daoism, there lie such relations in the human world or nature. To get free is to be fair and just.

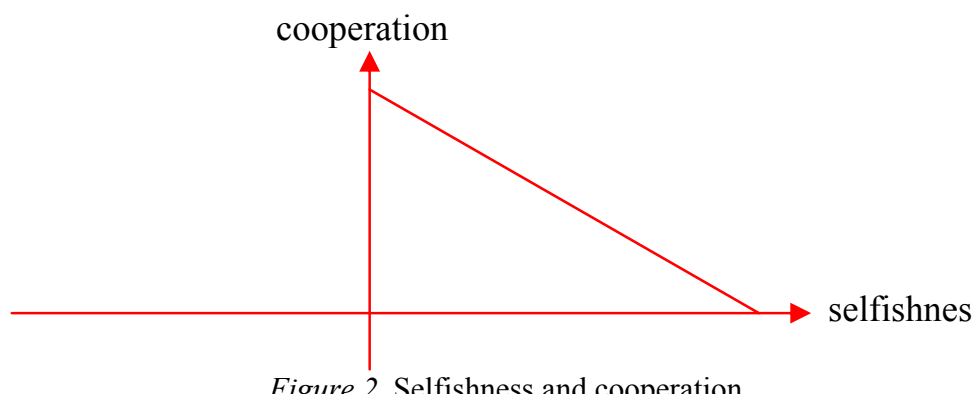

Decreasing function mechanism undergoes the link between selfness and cooperation. Human nature is selfness, or we can say cooperation, to some extent. The equilibrium is the harmony of these two variables. To be cooperative or to be selfish, that is the Dao, manifested in the $D e$.

They are just a couple of simple examples entailed in the myth of the $D D C$. As we know, $D D C$ was born to the governance of the state, stressing intervening as less as possible, with the focus on freedom and cooperation between the state and the subjected citizen. More typical ideas are to be interpreted.

We, normally, are familiar with such two-dimensional coordinate system. Plus, there are one-dimension, three-dimension, and polar schemes, which functions as well.

\section{DDC Under One-Dimensional Cartesian Coordinate}

One-dimensional coordinate system is an axis, which can reflect the flowing of Dao and De; Dao is going along with $D e$. Any point in the line, like a, b, c, d, ..., can be a reflection of De, underlying the Dao. Dao and $D e$ can be positive or negative, following the rule of the object is a plus (+), the opposite minus (-).

$\begin{array}{lllll}\mathrm{a} & \mathrm{b} & \mathrm{O} \cdot & \mathrm{c} & \text { d. } \\ \longrightarrow & & & \end{array}$

Figure 3. Dao and De.

Also, Dao can be viewed as the origin(O), on the right of which is yang, positive; the left yin, negative. Every object is a mixture of yin and yang, and there requires balance of them, whose imbalance state will create a tilt line. Generally, those which are moving violently, extroverted, ascending, warm, and bright are of yang; while those silent, introverted, descending, cold, and vague are of yin. In the light of heaven and earth, the light 
and clear qi of heaven bears the property of yang, whereas the heavy and unclear qi of earth of yin. In terms of water and fire, the cold water benefits the lower part, so that it is yin; fire carries the warmth and enhances the upper part, yang. To human body, head is of yang and feet yin; the outer of the body is yang and the guts are yin. $Q i$ is of yang and blood is of yin. The physical body is of yang and the mental is yin. The thoughts are yin and the actions are yang (Gan, 2016, p. 15). The interchanging of yin and yang is the dominant theme, just like the transfer of marks of plus (+) and minus (-). Yin-yang and yang-yin are on the move, which is very similar to our standing on the head instead of the feet.

What it matters is the unit to measure, a second, minute, hour, day, week, month, year, and so on, or we can say the $\operatorname{Dao}(D e)$ axis is unit-free. This can be an $H$ axis, a harmony of heaven(HA) and human beings(HB).

\section{$\mathrm{O} \cdot$}

Figure 4. Heaven and human beings.

Or an N one, nature, with which the Dao follows. It can be an integrity of $w u$ and you, Non-being and being, where $w u$ is negative, you is positive, its flowing from negative (left) to positive(right).

$\mathrm{O} \cdot$ $\mathrm{N} / \mathrm{D}$

Figure 5. Nature and Dao.

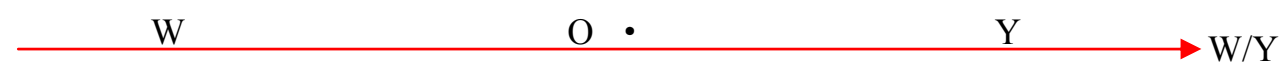

Figure 6. $W u$ and $y o u$.

In an certain interval $[\mathrm{a}, \mathrm{b}], y=f(x)=x-m, \quad \mathrm{x} \in D a o, \mathrm{y} \in D e, \mathrm{~m} \in \mathrm{R}$. The graph of the function is a curve; one's Dao and De is flowing forward and backward, up and down. In truth, Dao and De are equal; however, they can be in the inequalities.

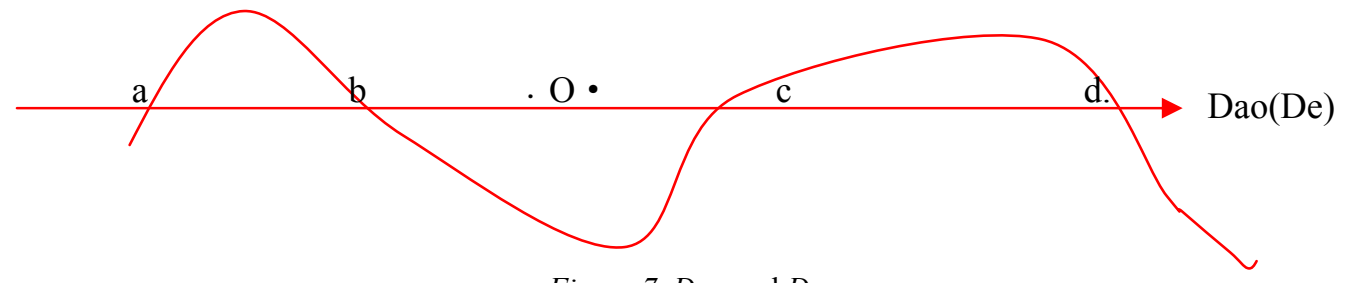

Figure 7. Dao and De.

Using Cartesian Coordinates we mark a point on a graph by how far along and how far up it is.... ${ }^{3}$ Every step or moving for the objects underlying the concepts above can be a point on the moving graph.

\section{DDC Under Two-Dimensional Cartesian Coordinates}

$D a o$ and De can be illustrated through the two-dimensional Cartesian coordinate system, where Dao is of $x$, and $D e$ is $y$. Superficially, $f(D a)=D(e)$, an increasing function, $D e$ going up with Dao. However, in truth, we can propose Dao $=|D e|, x=|y|(x \in D a o, y \in D e)$; but in functional expressions, generally it is expressed as follows: $\mathrm{y}=|x|,(\mathrm{x} \in D e, x \in \mathrm{R}, y \in D a o, y \geqq 0)$. De can be negative or positive, but Dao is always positive, it is an even function (see Figure 8). It is the same with nature(N) and Dao(D). $F(n)=D(a)$. Dao is entailed in

${ }^{3}$ Cartesian Coordinates, https://www.mathsisfun.com/data/cartesian-coordinates.html. 
nature, whose manifestation is $D e$. Nature is a set of all kinds of Daoes, whose growing and developing is the reflection of nature. Nature of an object is spontaneity, going as they like; it holds true to Dao (see Figure 9). $F(H B)=H A$, where human beings are integrity of the heaven (see Figure 10). $F(H B)=E$, where earth is the follower of the heaven (see Figure 11). Both of these two are increasing functions. $Y=f(w)=y o u, y o u, y$, is born of $w u$, being going together with non-being (see Figure 12). Yet, yin and yang are inverse functions; they are interdependent, either of which can stand for $x$, the other $y$. Yang $=f($ Yin $), y$ in $=f^{1}$ yang. Plus, (yang)yin and (yin)yang are also decreasing functions. Yin is increasing with the fall of yang, and vice versa. Yang is in the I and IV quadrants, and yin is in the II and III ones. The trip of an object implies the changing number of yin and yang. The weight of yang in I quadrant is equivalent to that of yin in the III one; it is the same policy with what is going on in the II and IV ones. If there is something wrong with the equation of yin and yang, there would be something imbalance to the object concerned (see Figure 13). Dao with nature, earth with heaven, are interlinked. Nature is of I quadrant, $(+,+)$, which is tangible, a leader of all. Heaven, of II, $(-,+)$, is negative, yin, or positive, yang; Dao, of III, is (-, -), intangible, underlying all, opposite or counterpart of nature; earth, of IV, $(+,-)$, opposing to heaven, has an intrinsic link with nature, positive in growing and negative nurturing (see Figure 14). In Figure 15, the common Dao is following the non-etynal Dao. All the things are changing; chaos dominates the life. Bending is to be straight; retreat is for further advancement. Bending and swirling will embrace an all-round knowledge. The horizontal axis, from negative to positive, shows the backward for forward; vertically, swirling instead of rocketing, circling complying with the growing rule (Xiao, 2013, p. 49).

Almost all the ideas or concepts can be expressed in such coordinate systems.

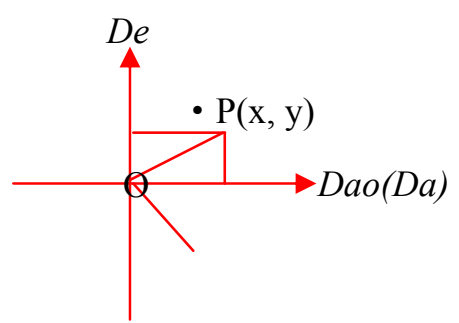

Figure 8. Dao and De.

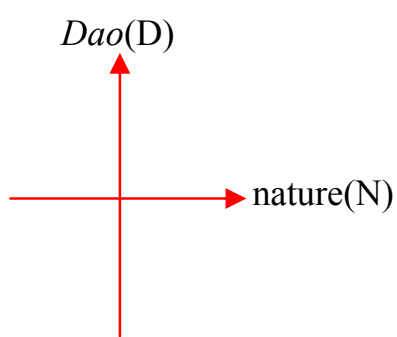

Figure 9. Nature and Dao.

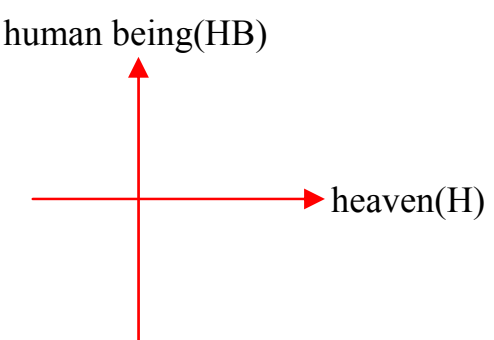

Figure 10. Heaven and human being.

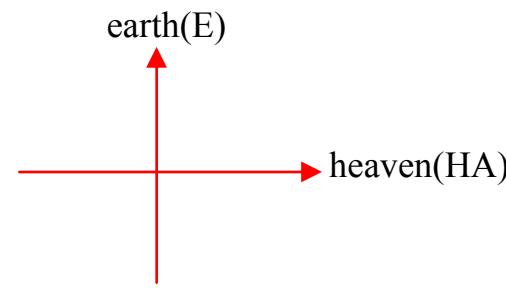

Figure 11. Heaven and earth.

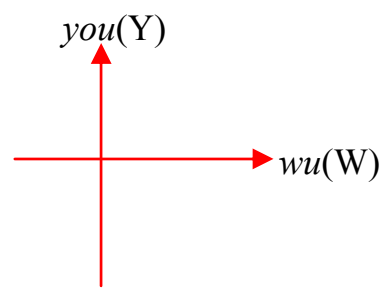

Figure 12.Wu and you.

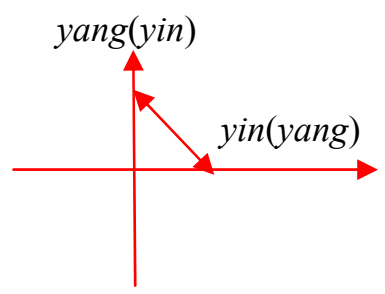

Figure 13. Yin and yang. 


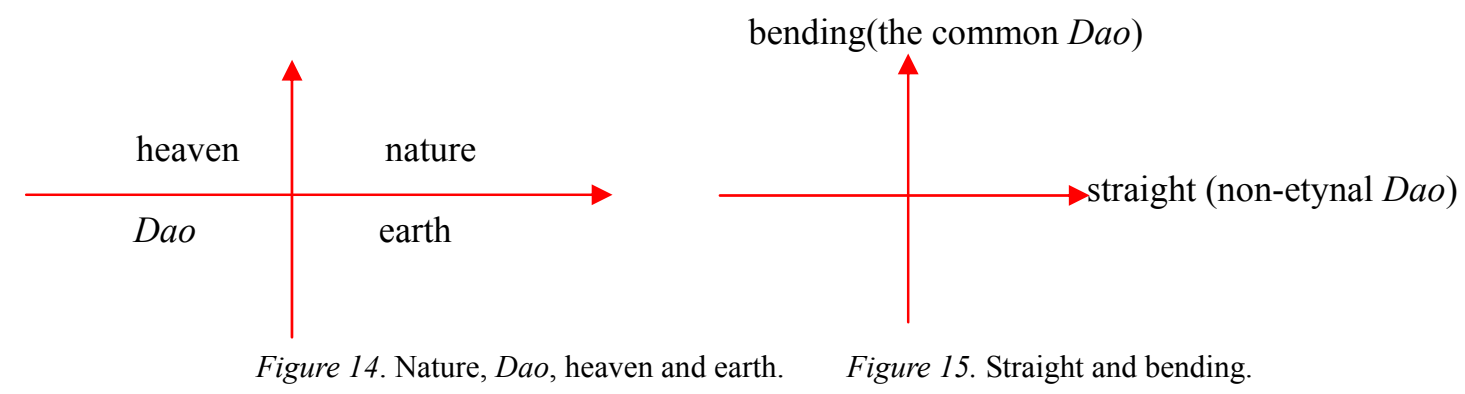

\section{DDC Under Three-Dimensional Cartesian Coordinates}

In three-dimensional space, there is a standard Cartesian coordinate system $(\mathrm{x}, \mathrm{y}, \mathrm{z})$. Start with a point which we call the origin, construct three mutually perpendicular axes, which we call the xx-axis, the yy-axis, and the zz-axis. With these axes any point $p p$ in space can be assigned three coordinates $p=(p 1, p 2, p 3)$. We can easily visualize two or three dimensions by drawing pictures of a plane or of space. If we represent a vector by a list of numbers, such as (a1, a2) $\in R 2(a 1, a 2) \in R 2$ and (a1, a2, a3) $\in R 3(a 1, a 2$, a3) $\in R 3$, we can easily go beyond three dimensions into four dimensions (a1, a2, a3, a4) $\in R 4(a 1, a 2$, a3, a4) $\in R 4$ or even to arbitrary dimension, such as nn-dimensions (a1, a2, .., an) $\in \mathrm{Rn}(\mathrm{a} 1, \mathrm{a} 2, \ldots$, an $) \in \mathrm{Rn}$, where $\mathrm{nn}$ is some positive integer. ${ }^{4}$

The three-dimensional scheme can be called the space coordinate. Three or more planes are pictured clearly the links between or among the variables. The gist of it is to abstain the changings from a point, a line into planes and space, of which points are the basic units. Lines, planes and spaces are sets of points. The vectors are introduced to the measurement of "products" in question. The ordered dimensions mirror the functions of vectors. All are of numbers, which suggests that we can order everything in the world.

Vectors enable us to measure the object with unit and direction, or employing the standard unit vectors to identify some relations. We can see a host of vectors in $D D C$, such as Dao and De, yin and yang, wu and you, dynamic and static, heaven and earth, and nature and human beings. All of these can be considered factors of space and time. Space and time are inseparable partners (Cole, 1999, p. 43). The cosmos in the Ancient Chinese term is the integration of time and space, including heaven and earth, the world, and the universe (Zhou, 2015, p. 30). The three-dimensional Cartesian coordinate will well demonstrate heaven, earth, human beings, or space, time, and all things, or Dao, yin (yang), all things. It holds true to nature, Dao and De.

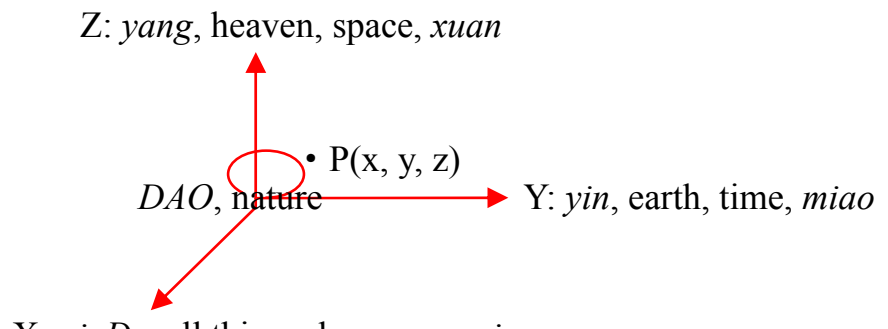

$\mathrm{X}: q i, D e$, all things, humans, wei

Figure 16. Right-handed coordinate system.

\begin{tabular}{l}
\hline $\begin{array}{l}\text { Vectors in two- and three- dimensional } \\
\text { https:/mathinsight.org/vectors_cartesian_coordinates_2d_3d. }\end{array}$
\end{tabular}




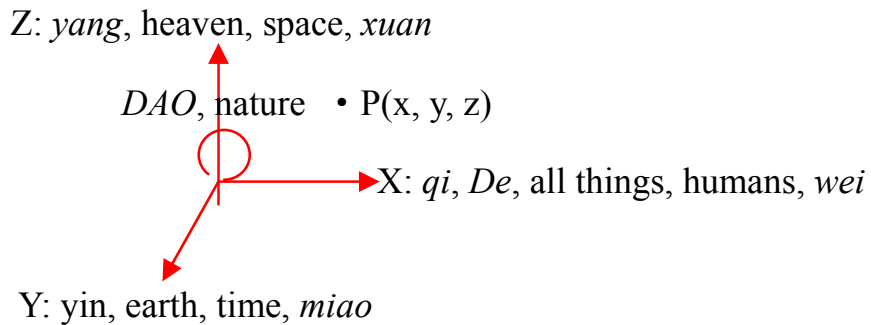

Figure 17. Left-handed coordinate system.

The three-dimensional coordinate systems, normally, takes axes $\mathrm{x}, \mathrm{y}$ as the independent variables, $\mathrm{z}$ being the dependent variable. On the other hand, $x$ can be the independent variable, $y$ and $z$ can be the dependent variables; this is to show that $x$ causes $y$ and $z$ arising. Or we can say, $y$ is the interdepent variable. It is intervening $x$ and $z$; such an intermediate variable links $x$ and $z$. Yet, what it matters in the real world is the pictures being more complex and complicated than $x, y$ and $z$. From this system, we can see xy, yz, zx planes and xyz space. In xy, there are Dao-De,Dao-heaven, time-space, subtle-mysterious (wei-miao) planes, in yz, yin-yang, heaven-earth, De-all things, human beings-space; in xz, Dao-earth, time-space, subtle-obstruse (miao-xuan), nature-all things. In xyz space, there are nature-Dao-De, time-space-human beings, subtle-mysterious-obstruse (wei-miao-xuan), heaven-earth-human beings.

Dao is the origin, in the center, following the nature, the whole picture of the space. It can be said that all are having their Dao, a follower of nature, of spontaneity, going on their own ways. To great extent, Dao and nature are identical. Also, these twins can be the core, the heart, the origin, all others turning around them. Qi (energy) gives birth to yin and yang, qi being the independent variable, yin and yang dependent variables. Point $\mathrm{P}$ can be established with $\mathrm{x} q i$, y yin, and $\mathrm{z}$ yang; three planes: yin qi, yang qi, and yin yang (see Figures 9 $\& 10)$.

\section{Polar Coordinates and DDC}

In mathematics, the polar coordinate system is a two-dimensional coordinate system in which each point on a plane is determined by a distance from a reference point and an angle from a reference direction.

The reference point (analogous to the origin of a Cartesian coordinate system) is called the pole, and the ray from the pole in the reference direction is the polar axis. The distance from the pole is called the radial coordinate, radial distance or simply radius, and the angle is called the angular coordinate, polar angle, or azimuth. $^{5}$

Of the $D D C$ polar coordinate system, $D a o$ is the reference point, $D e$ the radius, Ching the circle. $D D C$ is such a circle that myriads of things all are following the paths of such circles, and such circles are changing in shape and size. They are shrinking or expanding with the ups and downs of Dao and De. The angle, azimuth, can illustrate yin and yang with Figure 18. In the perspective of yin-yang theory, yin is the pole; yang is the radial coordinate, the varying distance between yang and yin carries the Dao and De. Five elements: metal, wood, water, fire, and earth are the contents of this theory. Metal is in the IV quadrant, wood I, fire II, earth III, and water is the pole(origin) (see Figure 18).

${ }^{5}$ Polar coordinate system, Wikipedia, https://en.wikipedia.org/wiki/Polar_coordinate_system. 


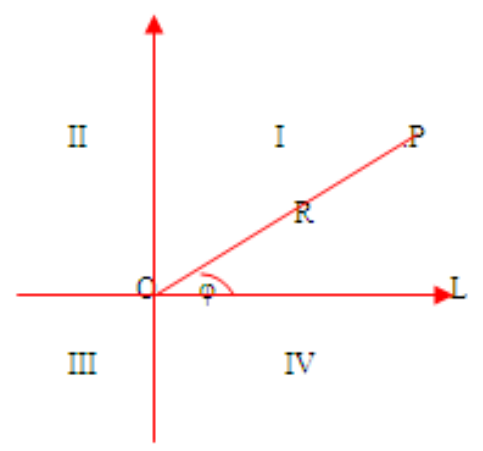

Figure 18. Dao, De, yin, yang, and five elements.

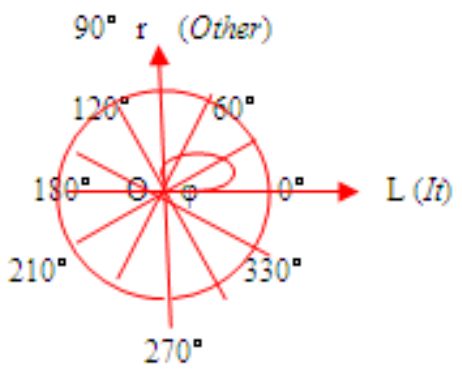

Figure 19. It and Other.

Are there really It and Other? Or really no It and Other? Where neither It nor Other finds its opposite is called the axis of the Way? When once the axis is found at the center of the circle there is no limit to responding with either, on the one hand, no limit to what is it, no limit to what is not. Therefore I say: "The best means is Illumination" (Cook, 2003, p. 26). "It" is "Other", and vice versa, which is of integrity. Non-being, wu, is the core, the origin, while It and Other are you, being, dancing with $I t$. It can be L, other being R, which is going along with L. $x=r \cos \theta y=\operatorname{rsin} \theta, x^{2}+y^{2}=r^{2}$ (see Figure 18).

The absolute quantity of a centripetal force is the measure of the same, proportional to the efficacy of the cause that propagates it from the centre, through the spaces round about (Newton, 2016, p. 5). The measurement of $D D C$ is to obtain the efficacy of the Dao causing all things pulled toward it, which is challenging the authority of the $D a o$, its calling and influences.

Moving force systems for the cosmos and Dao consists of three modes: explosion, linear, and circular (Xiao, 2013, p. 46). This can be expressed from the one-dimensional coordinates to two and three, then polar coordinate system. Taichiquan is one of the principal practices embracing the Daoist culture, whose essence owes to DDC. Doing Taichi is like following a helix. The hands, left or right, as the reference frame, angle of view, for each other. Also, both of them can be the angle of views for the feet or lower parts, and vice versa. The changing of hands or body turns are demonstrating the flows of yin and yang. Linear moving or curving derivatives are the components of the travelling of myriads of things.

“...there are individual and particular functions appropriate to each individual natural thing, as reasoning is to a human being, neighing to a horse, heating to a fire, and so on" (Haldane \& Ross, 1997, p. IX). There should be variety of functions to illustrate what is going on in $D D C$. Simplified patterns or complicated ones all will reveal the brilliance and complexity of Daoism. Graphs and numbers analysis will enhance the 
understanding and recognizing of the poetic language.

\section{Concluding Remarks}

The Daoes of heaven, earth, and human beings are composites of the cosmos. The marriage of yin and yang, heaven and earth, space and time, Dao and De, and light and dust recognizes a challenging and instructive project based on the coordinate systems. These first sketches about $D D C$ may invite more arguments; however, they can be candidates to the interpreting of such a myth. As we know, the Chinese civilization features as the application of image (xiang) and numbers (Xiao, 2013, p. 548). Luckily, the coordinate systems well coordinate the graph and number. The functional point-line-plane-space analysis of the ordered pairs concisely identifies the feature and nature of the key and critical terms of the $D D C$. Yet, if it goes with data analysis, introducing some measurement units, the initiative will render more substantial justice.

The first inkling of maths and myth of $D D C$ is an attempt to explore something invisible to us in the cosmos. This is a small step to embrace the idea that $D D C$ can be mathematicalized. Nature and Nature's laws lay hid in night, God said, Let $D D C$ be! And all was light (Hawking \& Mlodinow, 2010, p. 39). Laozi and Zhuangzi were knights, making us flight. Math is of might, dissecting it right. $D D C$ 's sight is tight with math's might. In this light, there will be more sights about the functional reflection of this poetic might.

\section{References}

Cole, K. C. (1999). The universe and teacup: The mathematics of truth and beauty. New York: A Harvest Book Harcourt Inc.

Cook, S. (Ed.). (2003). Hiding the world in the world: Uneven discourses on the Zhuangze. Albany, N.Y.: State University of New York Press.

Gan, Y. (2016). Yi's arguments of Dao De Ching. Beijing: Standards Press of China.

Haldane, E. S., \& Ross, G. R. T. (Trans.). (1997). Descartes-Key philosophical writings. UK: Wordsworth Classics of World Literature.

Hawking, S., \& Mlodinow, L. (2010). The grand design. London: Bantam Books.

Newton, I. (2016). Mathematical principles of natural philosophy. Beijing: Higher Education Press.

Stewart, J. (2016). Calculus (6th ed.). China: China Renmin University Press.

Xiao, Q. G. (2013). The Dao of heaven from Laozi. Beijing: Kyushu Publishing House.

Zhang, R. J. (2017). Mathematical ideas and cultures. Beijing: Science Press.

Zhou, F. (2015). The Dao of Nature from Laozi and Zhuangzi. Chuangchun: Jinlin People's Publishing House.

Zhu, H. W., \& Cheng, H. B. (2018). Functions and functional thoughts. Beijing: Science and Technology of China Press. 